Article

\title{
Investigation of Bus Drivers' Reaction to ADAS Warning System: Application of the Gaussian Mixed Model
}

\author{
Wei Ye ${ }^{1,2,3}$, Yueru Xu ${ }^{1,2,3,4, *}$, Feixiang Zhou ${ }^{1,2,3}$, Xiaomeng Shi ${ }^{1,2,3}$ and Zhirui Ye ${ }^{1,2,3}$ \\ 1 Jiangsu Key Laboratory of Urban ITS, Southeast University, Nanjing 211189, China; \\ 213180148@seu.edu.cn (W.Y.); 213181575@seu.edu.cn (F.Z.); shixiaomeng@seu.edu.cn (X.S.); \\ yezhirui@seu.edu.cn (Z.Y.) \\ 2 Jiangsu Province Collaborative Innovation Center of Modern Urban Traffic Technologies, \\ Southeast University, Nanjing 211189, China \\ 3 School of Transportation, Southeast University, Nanjing 211189, China \\ 4 Intelligent Transportation System Research Center, Southeast University, Nanjing 211189, China \\ * Correspondence: xuyr@seu.edu.cn
}

Citation: Ye, W.; Xu, Y.; Zhou, F.; Shi, X.; Ye, Z. Investigation of Bus Drivers' Reaction to ADAS Warning System: Application of the Gaussian Mixed Model. Sustainability 2021, 13, 8759. https://doi.org/10.3390/su13168759

Academic Editors: Feng Zhu,

Wenbo Zhang, Yuntao Guo and Jian Wang

Received: 31 May 2021

Accepted: 2 August 2021

Published: 5 August 2021

Publisher's Note: MDPI stays neutral with regard to jurisdictional claims in published maps and institutional affiliations.

Copyright: (c) 2021 by the authors. Licensee MDPI, Basel, Switzerland. This article is an open access article distributed under the terms and conditions of the Creative Commons Attribution (CC BY) license (https:// creativecommons.org/licenses/by/ $4.0 /)$.

\begin{abstract}
Road crashes cause serious loss of life and property. Among all vehicles, buses are more likely to encounter crashes. In recent years, the advanced driving assistance system (ADAS) has been widely used in buses to improve safety. The warning system is one of the key functions and has proven effective in reducing crashes. However, drivers often ignore or overreact to ADAS warnings during naturalistic driving scenarios. Therefore, reactions of bus drivers to warnings need further investigation. In this study, bus drivers' responses to lane departure warning (LDW) and forward collision warning (FCW) were investigated using 20-day naturalistic driving data. These reactions could be classified into three categories, namely positive, negative, and overreaction or emergency, by employing the Gaussian mixture model. The authors constructed a framework to quantify drivers' reactions to the warning and study the reaction characteristics in different environments. The results indicate that drivers' reactions to FCW were more positive than to LDW, drivers reacted more positively to LDW and FCW while driving on highways than on urban roads, and drivers reacted more positively at night to LDW and FCW than during daytime. This study gives support to an adaptive ADAS considering varying bus driver characteristics and environments.
\end{abstract}

Keywords: advanced driver assistance systems; forward collision warning; lane departure warning; bus drivers' reaction; Gaussian mixed model

\section{Introduction}

With the increasing number of vehicles, road safety is attracting more attention all over the world. According to the report of the World Health Organization in 2018, road traffic crashes cause 1.35 million deaths every year and is the ninth largest cause of human death. By 2030, road crashes are expected to increase from the ninth factor of mortality to the seventh, accounting for approximately 1.8 million deaths per year [1]. Road crashes cause serious loss of life and property. Among all vehicles, buses are more likely to encounter crashes. In China, from 2009 to 2013, there were 14,000 road traffic crashes caused by buses, resulting in 3500 deaths and 16,000 injuries. The number of crashes and deaths account for about $1 \%$ of the total number of road traffic crashes in China but the number of buses only accounts for $0.2 \%$ of the total number of motor vehicles in China [2]. Therefore, bus safety should be investigated to improve road safety.

Various researchers have focused on exploring the characteristics of road crashes [3,4] and tried to improve road safety [5,6]. After the investigation, approximately $90 \%$ of road crashes were found to be caused by human factors [7]. With the development of industrial engineering and information technology, various advanced driver assistance systems (ADAS) have been developed to improve drivers' behavior and facilitate safe 
driving. These systems mainly include adaptive cruise control (ACC) [8], autonomous emergency braking (AEB) [9], driver monitoring system (DMS) [10], forward collision warning (FCW) [11], lane departure warning (LDW) [12], etc. ADAS has been promoted worldwide and has been proven to play a significant role in preventing traffic crashes [13], reducing emissions [14] and improving the driving experience [8]. In China, many buses have also installed several ADAS functions to help improve driver behavior and reduce safety risks.

At present, the two most widely used functions are FCW and LDW. FCWs and LDWs have been reported to reduce crashes by $16.3 \%$ on freeways and $11.6 \%$ on other roads [15]. LDW can improve the driver's lateral driving behavior. After adopting LDW, unconscious lane departure reduced by $30-35 \%$ [16]. Meanwhile, FCW is mainly used to enhance drivers' longitudinal driving behavior. According to the research of Birrell, S.A. et al. in 2014 [12], after using FCW, the mean headway increases to $2.3 \mathrm{~s}$ and time spent traveling closer than $1.5 \mathrm{~s}$ to the vehicle in front decreases nearly three times.

However, the application of LDW and FCW systems also result in some side effects. The long-term use of the ADAS has a tendency to reduce the drivers' perception of road risks [17] and weaken their ability to address emergencies [18]. In addition, LDW, FCW, and some other warning systems cannot control the mobility of the vehicle. The actual effect of these warnings is based on drivers' reactions, requiring active driver implication, which is affected by the drivers' trust on the warning system [19], secondary tasks [20], and environments [21].

In addition, for different drivers, the characteristics of car-following behavior and cornering behavior are different [22,23], and the adoption of uniform ADAS standards may lead to the decline of driver acceptance. Therefore, different drivers' reactions to the ADAS warnings and the different reactions under different environments need to be further investigated. A better understanding of drivers' reactions to LDW and FCW systems will facilitate the development of an adaptive ADAS.

Researchers have put in great effort regarding studying drivers' reaction to LDW and FCW systems. For instance, driving simulators have been widely applied [24,25] due to their low cost, safety, ease to use, and ability to switch to multiple traffic environments in a short time. However, former findings suggest that performance in driving simulators was not a strong predictor of performance in the on-road assessment [26]. Thus, drivers reactions could be different when interacting with an ADAS through the simulator and in real-world conditions. Another common approach is to conduct field operational tests (FOTs) [21,27]. This method can capture drivers' behaviors more realistically than simulators [28]. An FOT performed in Wuhan, China, revealed that driver acceptance of the FCW function was considerably higher than that of the LDW function, and the acceptance on expressways and freeways was significantly higher than on urban roads [27]. However, it is difficult to study the drivers' reactions at different times or under different weather conditions because of the short span of the FOT. In addition, drivers may lose their spontaneity when they are aware that their driving is being monitored. Therefore, to overcome the limitations of simulator-based studies and FOTs, naturalistic driving studies (NDS) have emerged as a better method for evaluating driving behavior [29-31]. Naturalistic driving is a research method that provides insight into everyday driver behavior by recording details of the driver, the vehicle, and the surroundings through unobtrusive data-gathering equipment and without experimental control [32]. Using naturalistic driving data to study drivers' reactions can obtain more diversified and realistic insight into driving behavior, which can better support and supplement the previous studies. Julia Orlovska discussed the effects of the driving context on the usage of the ADAS and stressed the importance of the driving context parameters in the design, development, and evaluation of ADAS [33], which added new information in the field of human-machine interactions with ADAS.

Moreover, in a previous study, the main research object was private cars [34-36]. There is little research focusing on bus drivers, considering a bus is different from private cars in terms of braking performance, turning ability, and control mode. Therefore, a bus driver's 
reaction to an ADAS warning may be different from a car driver's reaction, which needs to be further investigated.

This study analyses bus drivers' reactions to LDW and FCW systems based on the naturalistic driving data. The difference in the drivers' reactions at different times and different environments were investigated. The main contributions of this study are as follows:

1. In this study, a quantitative framework of bus drivers' reactions to ADAS is constructed based on the unsupervised method and fuzzy logic method, and this framework can be transferred to other relative studies, thereby introducing a new perspective for the study of drivers' acceptance of the ADAS.

2. This study investigates the reaction of bus drivers to LDW and FCW, which is rare in previous studies. This research can support the design of bus adaptive ADAS that can improve bus drivers' acceptance and therefore lead to better safety outcomes.

3. This study investigates the difference in driver responses to ADAS warnings between daytime and nighttime, which has not yet been comprehensively understood due to its long time-span.

The remaining sections of this paper are structured as follows. Section 2 identifies the sources of the naturalistic driving data and methods (Gaussian mixed model and membership degree) applied in the paper. In Section 3, the result of Gaussian mixed model is given and explained. Based on this result, the authors investigated bus drivers' overall reaction to LDW, FCW, and reaction in different environments. In Section 4, the study is discussed in light of previous studies. The limitations and future scope of the study are also stated.

\section{Materials and Methods}

The key objectives of this study were to explore bus drivers' reactions to LDW and FCW systems with naturalistic driving data and to discover reaction differences under different times and environments. In order to investigate this problem, the authors developed a framework for harnessing naturalistic driving data generated by ADAS. Reactions were classified with an unsupervised learning method and drivers' reactions to warnings were quantified based on the concept of membership degree (Figure 1).

The proposed framework consists of three major steps: (1) data acquisition, (2) data pre-processing, and (3) warning reaction classification. Herein, the warnings of the LDW and FCW alone are discussed, and the other warning types (such as headway monitoring warning) and other kinds of drivers (such as drivers of cars) can be processed in the same manner. 


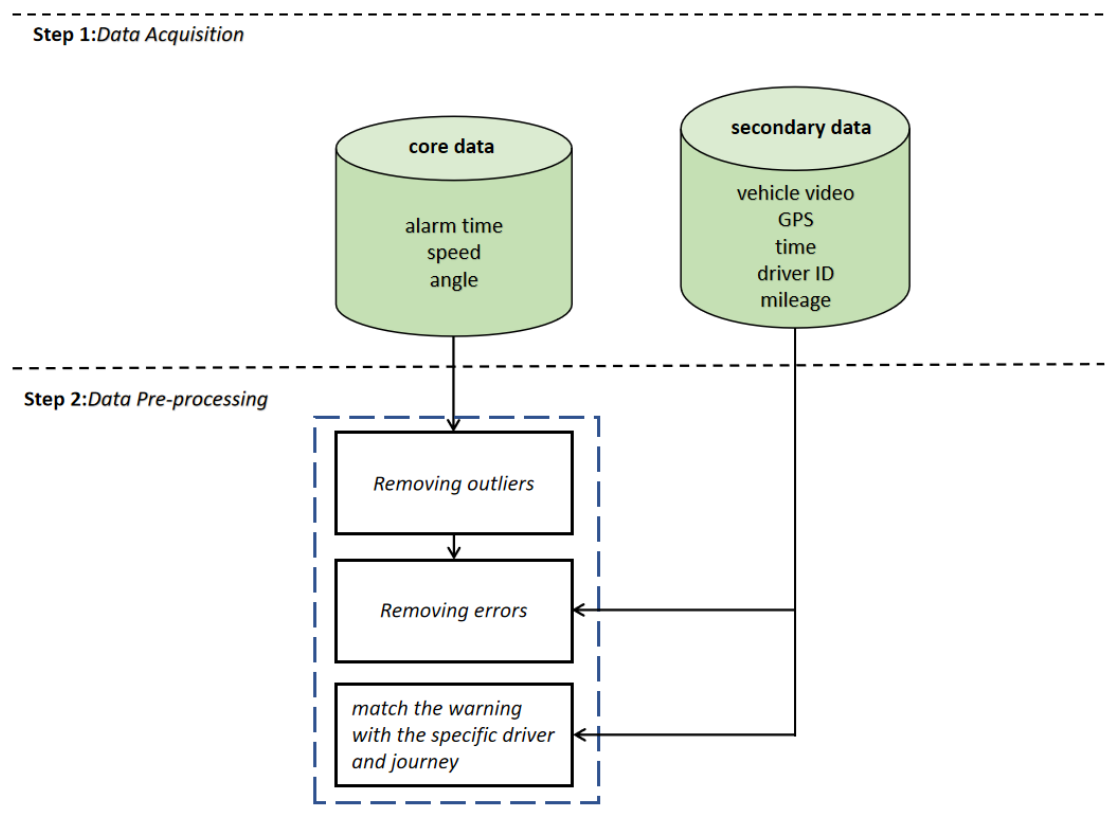

Step 3:Warning Reaction Classification

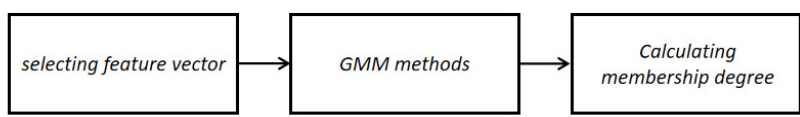

Figure 1. Methodological approach of the study.

\subsection{Data Acquisition and Pre-Processing}

The driving data utilized in this study were from the intelligent data platform of bus in the Jiangsu province, which was set up by the Jiangsu provincial government. Most commercial vehicles in Jiangsu have installed ADAS, including various warning functions. The intelligent data platform collects naturalistic driving and warning data from these buses. All drivers in the platform have been informed and agreed in advance that their driving information (videos, GPS, driving characteristics, etc.) and personal information (gender, age, identity card information, and driving license information) will be collected and used for relevant research purposes. In this study, 20 buses were selected and all of them were using the Mobileye M630 system, which is a mature system and has been used in many other studies [37]. The equipment applied in the buses involved is illustrated in Figure 2.

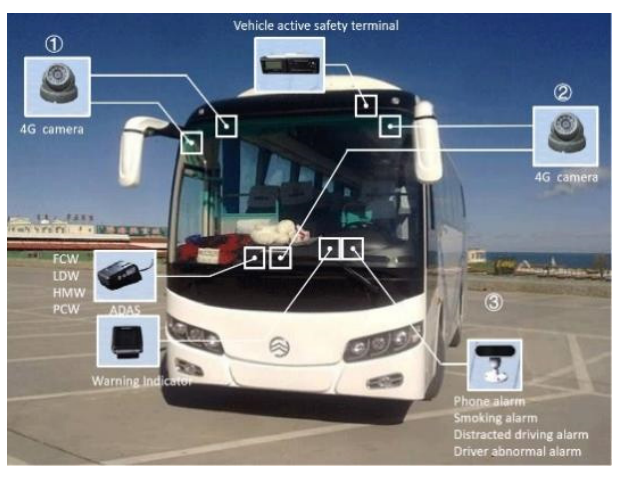

Figure 2. Equipment. 
Warning data from the platform contains nine dimensions, including the environmental information (latitude, longitude, altitude, and time), vehicle running conditions (speed and angle), driver information (age and mileage), and alarm duration. Moreover, video information was also available. The entire process from the onset to the end of the alarm was recorded in the videos.

In our study, we used ADAS data of 20 drivers during 20 days of driving, which contains more than 13,000 LDW and FCW events. Drivers involved are between 35 and 50 years old (mean $=42.35, \mathrm{SD}=4.209$ ). Considering that bus drivers are mainly experienced male drivers and their experience may affect the reaction to ADAS [38], the samples selected in this study are all male drivers with more than 10 months of experience.

To study the characteristics of the drivers' responses to ADAS in different road environments, the road environment needs to be assessed for each alarm. The driving course information of the 20 drivers captured by the GPS is depicted in Figure 3. The GPS data were processed with Map API of AutoNavi and the road types for each alarm position were obtained. The road types were classified into the following three categories: freeways, urban roads, and other road types (such as ramps and rural roads).

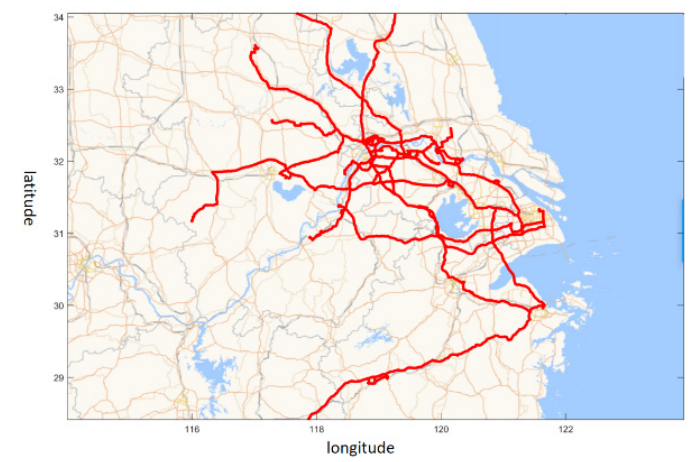

Figure 3. Driving course.

\subsection{Selection of Feature Vectors}

The input of the reaction classification model was assumed to be x. For LDW and FCW, the input $x$ was different. In this study, alarm duration was selected as one input to evaluate the drivers' reaction type. Alarm duration refers to the time from the onset to the end of the ADAS alarm. Long alarm duration means that drivers cannot address the alarm on time, which is always a negative reaction and may lead to dangerous events. Moreover, the core function of ADAS is improving safety. From the perspective of traffic safety, the change of speed or angle should also be considered. Numerous research studies have proven that crash rates increase as speed or steering angle variation increases [39-41]. Previous studies have shown that LDW mainly affects the drivers' lateral driving behavior [16], while FCW mainly affects the drivers' longitudinal driving behavior [12]. In the present paper, the change of speed and angle refers to the change of speed and angle from the beginning of the alarm to the end of the alarm. The authors considered the change in the drivers' steering angle to study the drivers' response to LDW and the change in the drivers' driving speed to study the drivers' response to FCW. Therefore, for LDW, the alarm time duration and change in angle were considered as the input feature vector, while for FCW, the alarm time duration and change in speed were selected as the input vector. All the variables used for classification are depicted in Figure 4. 


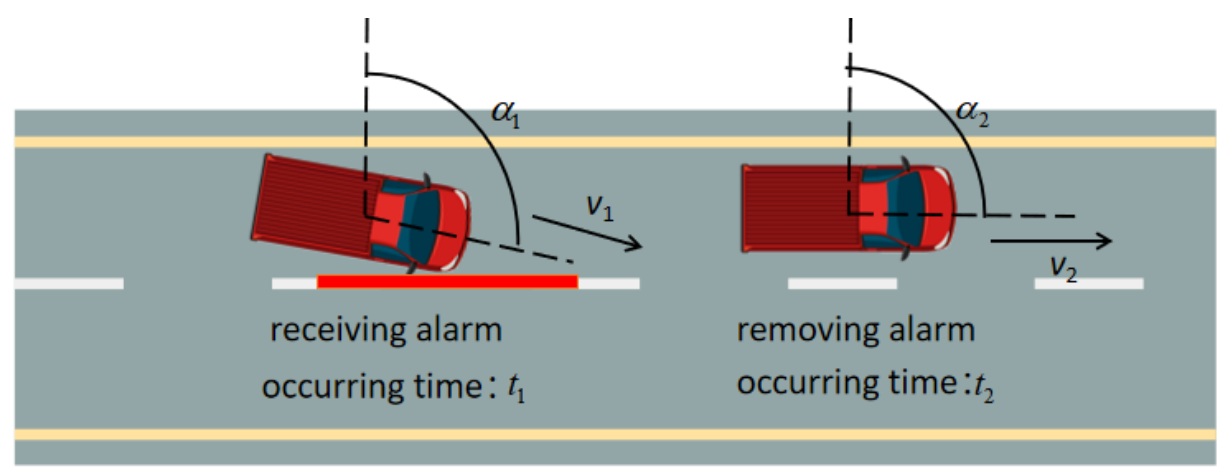

Figure 4. Variables used for classification.

\subsection{Classification of Drivers' Reactions}

After selecting the quantitative feature vector of the reaction behavior, the appropriate method to classify the reaction behavior was selected. The data characteristics of speed change, angle change, and alarm time (such as mean value and standard deviation) are not directly used for assessment because the method is not resistant to abnormal values and cannot reflect the distribution characteristics of the driver response behavior. For the data-based driving behavior classification problem, the commonly used classification methods for driving behavior are unsupervised clustering (such as k-means) [42], latent Dirichlet allocation (LDA) [43], and the support vector machine (SVM) [44]. Considering that the LDA requires semantic features, the SVM requires labels, and they only function effectively in the case of binary classification, using the clustering method is an appropriate choice. In this study, the Gaussian mixed model (GMM) method was selected. Furthermore, the output of the GMM is a probability vector that has an improved effect on the boundary of the classification region and can retain more information. Moreover, the k-means method is sensitive to outliers and noise points that may lead to the lack of interpretability of the clustering results.

\subsubsection{GMM}

GMM is a linear combination of several Gaussian distribution functions. The expression of a multi-dimensional Gaussian distribution is

$$
p(x, \mu, \Sigma)=\frac{1}{(2 \pi)^{\pi / 2}|\Sigma|^{1 / 2}} \exp \left(-\frac{1}{2}(x-\mu)^{T} \Sigma^{-1}(x-\mu)\right)
$$

Here, $\mu$ denotes the mean vector of the Gaussian distribution. $\sum$ denotes the covariance matrix that is used to control the shape of the Gaussian distribution. The form of $\sum$ is defined below:

$$
\Sigma=\left[\begin{array}{cc}
E\left[\left(X_{1}-\mu_{1}\right)\left(X_{1}-\mu_{1}\right)\right] E\left[\left(X_{1}-\mu_{1}\right)\left(X_{2}-\mu_{2}\right)\right] \ldots E\left[\left(X_{1}-\mu_{1}\right)\left(X_{n}-\mu_{n}\right)\right] \\
E\left[\left(X_{2}-\mu_{2}\right)\left(X_{1}-\mu_{1}\right)\right] E\left[E\left(X_{2}-\mu_{2}\right)\left(X_{2}-\mu_{2}\right)\right] \ldots E\left[\left(X_{2}-\mu_{2}\right)\left(X_{n}-\mu_{n}\right)\right] \\
\vdots \\
\vdots \\
E\left[\left(X_{n}-\mu_{n}\right)\left(X_{1}-\mu_{1}\right)\right] E\left[E\left(X_{n}-\mu_{n}\right)\left(X_{2}-\mu_{2}\right)\right] \ldots E\left[\left(X_{n}-\mu_{n}\right)\left(X_{n}-\mu_{n}\right)\right]
\end{array}\right]
$$

The form of the Gaussian mixture model is shown below:

$$
p(x)=\sum_{k=1}^{K} \pi_{k} N\left(x \mid \mu_{k}, \Sigma_{k}\right)
$$


where $N\left(x \mid \mu_{k}, \Sigma_{k}\right)$ is the $k$ th component in the Gaussian mixture model, $K$ denotes the number of Gaussian distributions, and $\pi_{k}$ represents the coefficient of the component that fulfills the following rules:

$$
\begin{aligned}
& \sum_{k=1}^{K} \pi_{k}=1 \\
& 0<\pi_{k} \leq 1
\end{aligned}
$$

$\pi_{k}$, as mentioned above, can be regarded as the probability that the $k$ th class is selected. A new $K$-dimensional random variable $\mathrm{z}$ is introduced here. $z_{k}$ can only assume two values, 0 or $1 . z_{k}=1$ represents the probability that the $k$ th class is selected, implying $p\left(z_{k}=1\right)=\pi_{k}$; that is,

$$
\begin{gathered}
z_{k} \in\{0,1\} \\
\sum_{K} z_{k}=1
\end{gathered}
$$

Assuming that $z_{k}$ is independent and identically distributed, the joint probability distribution of $\mathrm{z}$ can be obtained as follows:

$$
p(z)=p\left(z_{1}\right) p\left(z_{2}\right) \ldots p\left(z_{k}\right)=\prod_{k=1}^{K} \pi_{k}^{z_{k}}
$$

The data in the original problem can be classified into several categories. Evidently, the data in each category obey the Gaussian distribution. This statement can be expressed based on conditional probability as follows:

$$
p\left(x \mid z_{k}=1\right)=N\left(x \mid \mu_{k}, \Sigma_{k}\right)
$$

In other words, the data in category $k$ obey the Gaussian distribution. Therefore, the above formula can be written as follows:

$$
p(x \mid z)=\prod_{k=1}^{k} N\left(x \mid \mu_{k}, \Sigma_{k}\right)^{z_{k}}
$$

According to the total probability formula, it can be concluded that

$$
p(x) \sum_{z} p(z) p(x \mid z)=\sum_{k=1}^{K} N\left(x \mid \mu_{k}, \Sigma_{k}\right)
$$

$\gamma\left(z_{k}\right)$ is set as a posterior probability of the $k$ th component, according to the Bayesian formula:

$$
\gamma\left(z_{k}\right)=p\left(z_{k}=1 \mid x\right)=\frac{p\left(z_{k}=1\right) p\left(x \mid z_{k}=1\right)}{p\left(x, z_{k}=1\right)}=\frac{\pi_{k} N\left(x \mid \mu_{k}, \sum_{k}\right)}{\sum_{j=1}^{K} \pi_{j} N\left(x \mid \mu_{j}, \Sigma_{j}\right)}
$$

and the posterior probability is solved based on the expectation-maximization (EM) algorithm.

\subsubsection{EM Algorithm}

EM algorithm is a solution for determining the maximum likelihood estimation or the maximum a posteriori estimation of parameters in the probability model, where the probability model depends on unobservable hidden variables. In the process of using the EM algorithm to solve Gaussian mixture distribution parameters, the unobservable hidden variable is $z_{k}$, as mentioned above. Suppose that the dataset to be classified is 
$x=\left\{\mathrm{x}_{1}, \mathrm{x}_{2}, \mathrm{x}_{3}, \ldots \mathrm{x}_{\mathrm{N}}\right\}$ and $N$ is the number of vector data. In the GMM model, there are three parameters to be estimated: $\pi, \boldsymbol{\mu}$, and $\sum$. According to Equation (3),

$$
p\left(x \mid \pi, \mu, \sum\right)=\sum_{k=1}^{K} \pi_{k} N\left(x \mid \mu_{k}, \Sigma_{k}\right)
$$

To estimate these three parameters, the maximum likelihood functions of these three parameters are required to be solved. Consider $\mu$ be the differential in the above formula and consider the derivative function to be equal to zero. The maximum likelihood estimation of each parameter is obtained, where the mean vector is

$$
\mu_{k}=\frac{1}{N_{k}} \sum_{n=1}^{N} \gamma\left(z_{n k}\right) x_{n}
$$

$\gamma\left(z_{n k}\right)$ denotes the posterior probability that point $x_{n}$ belongs to cluster $k$ which is calculated in Equation (12) with the initial values of $\pi, \mu$, and $\sum$, or $\pi, \mu$, and $\sum$ as obtained in the previous iteration. Accordingly,

$$
N_{k}=\sum_{n=1}^{N} \gamma\left(z_{n k}\right)
$$

The covariance matrix and mixture coefficient are obtained as shown below:

$$
\begin{gathered}
\sum_{k}=\frac{1}{N_{k}} \sum_{n=1}^{N} \gamma\left(z_{n k}\right)\left(x_{n}-\mu_{k}\right)\left(x_{n}-\mu_{k}\right)^{T} \\
\pi_{k}=\frac{N_{k}}{N}
\end{gathered}
$$

After solving Equations (14), (16) and (17), the new values of $\pi_{\mathrm{k}}, \mu_{\mathrm{k}}$, and $\sum_{k}$ can be obtained and these values can be inserted into Equation (13) to progress to the next iteration.

\subsection{Drivers' Membership Degree to Reaction Types}

As depicted in Figure 5, there is a significant difference between Driver 8 and Driver 11 in the distributions of their reactions to LDW and FCW. To describe this difference, in this section, the membership degree is introduced to express the drivers' reactions.

The membership degree is an important concept in fuzzy mathematics that can be applied in the recognition of driving behavior [45] and the driver assistance system [46].

Supposing that $A_{i}(x) \in[0,1]$, corresponding to any element $x$ on the set $U, A_{i}$ is called a fuzzy set on $U$ and $A_{i}(x)$ is called the membership of $x$ to $A$. When $x$ changes in $U, A_{i}(x)$ represents a function that is called the membership function of $A_{i}$. The closer the membership degree $A_{i}(x)$ is to 1 , the higher the degree that $x$ belongs to $A_{i}$. The closer the membership degree $A_{i}(x)$ is to 0 , the lower the degree that $x$ belongs to $A_{i}$. For an input $\mathrm{x}$, the membership degree of the input to different categories can be obtained. Suppose that the number of categories is $j$ and the membership degree vector to every input $x$ is $Y(x)=\left(A_{1}(x), A_{2}(x), \ldots, A_{j}(x)\right)$.In general, the selection of the membership function $A_{i}(x)(i=1,2 \ldots j)$ should meet the following rule:

$$
\sum_{i=1}^{j} A_{i}(x)=1
$$



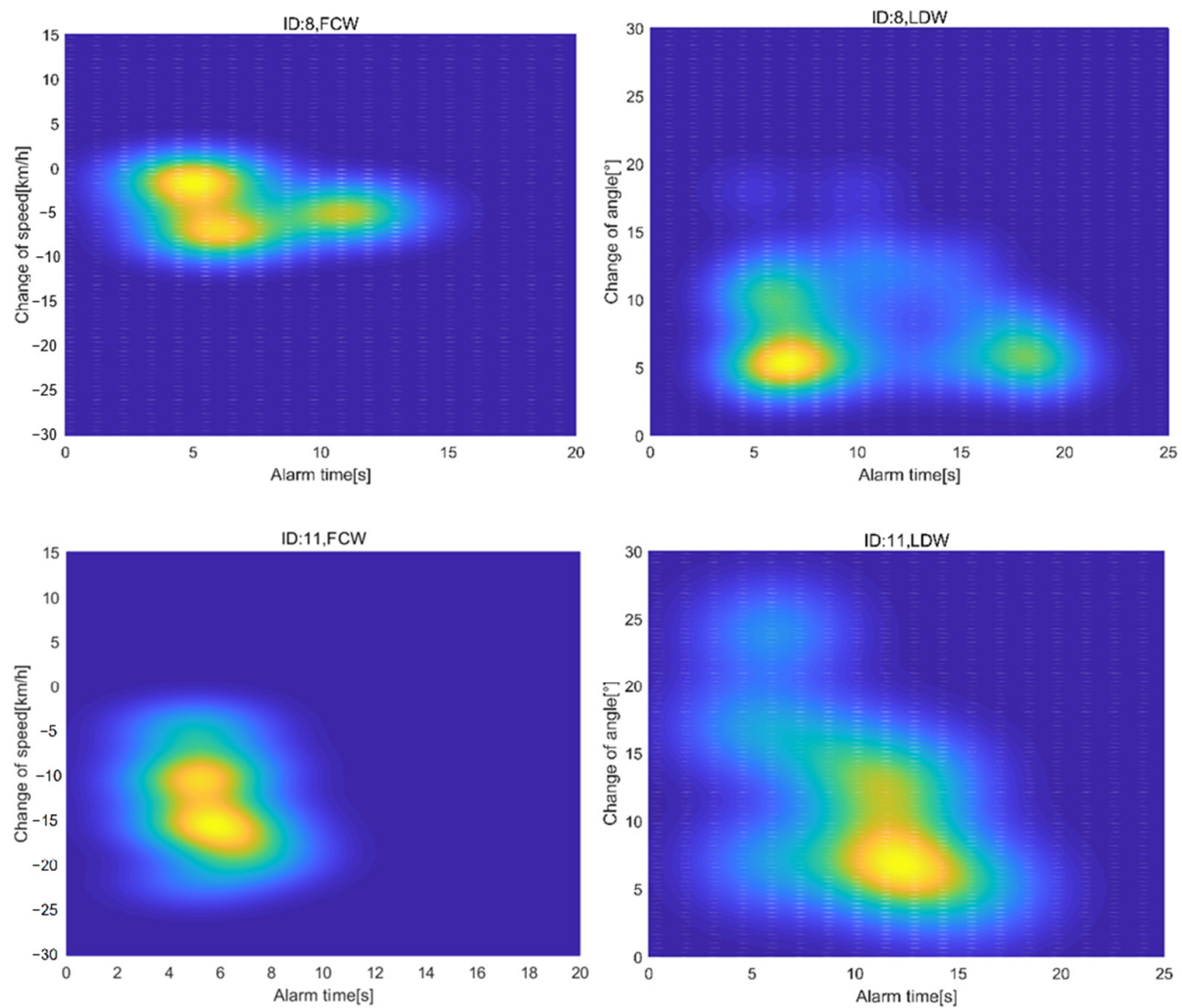

Figure 5. Difference in the distribution of reactions among different drivers.

Here, for each input response data $x$, its membership degree to category $j$ is equal to the probability that it belongs to $N\left(x \mid \mu_{j}, \Sigma_{j}\right)$,according to Equation (12):

$$
p\left(z_{j}=1 \mid x\right)=\frac{\pi_{j} N\left(x \mid \mu_{j}, \sum_{j}\right)}{\sum_{i=1}^{K} \pi_{i} N\left(x \mid \mu_{i}, \sum_{i}\right)}
$$

For a driver, the average of the membership degree of all his reactions to category $j$ is used to express the membership of the driver to category $j$. The membership degree of a driver can be calculated as shown below:

$$
\begin{gathered}
M(i)_{\_} L D W_{j}=\frac{1}{K_{i}} \sum_{x \in A_{i}} \frac{\pi_{j} N\left(x \mid \mu_{j}, \Sigma_{j}\right)}{\sum_{k=1}^{N} \pi_{k} N\left(x \mid \mu_{k}, \Sigma_{k}\right)} \times 100 \%(j=1,2,3, \ldots N ; i=1,2, \ldots, 20) \\
M(i)_{-} F C W_{j}=\frac{1}{K_{i}} \sum_{x \in A_{i}} \frac{\pi_{j} N\left(x \mid \mu_{j}, \Sigma_{j}\right)}{\sum_{k=1}^{N} \pi_{k} N\left(x \mid \mu_{k}, \Sigma_{k}\right)} \times 100 \%(j=1,2,3, \ldots, N ; i=1,2, \ldots, 20)
\end{gathered}
$$

Here, $j$ denotes the category of reactions and $N$ denotes the number of reaction clusters. $i$ represents the ID of the driver. $A_{i}$ denotes the set of all the warning reactions and $K_{i}$ denotes the number of warning reactions. $M(i)_{-} L D W_{j}$ represents the membership degree of driver $i$ to reaction category $j$ of $L D W$ and $M(i)_{-} F C W_{j}$ represents the membership degree of driver $i$ to reaction category $j$ of $F C W$. The membership degree can be used to identify the drivers' usual reaction to warnings. 


\subsection{Statistical Characteristics of the Membership Degree}

To study the drivers' response to ADAS warning, the drivers' overall situation and differences among the drivers can be evaluated based on statistical characteristics.

For the measurement of the overall situation of the sample, the most commonly used approach is to calculate the average $\left(M_{\text {mean }} L D W_{j}\right.$ and $\left.M_{\text {mean }} F C W_{j}\right)$. This measure can be calculated as shown below:

$$
\begin{gathered}
M_{\text {mean_ } \_D W_{j}}=\frac{1}{N} \sum_{i=1}^{N} M(i)_{-} L D W_{j}(j=1,2,3, \ldots, N) \\
M_{\text {mean_}} F C W_{j}=\frac{1}{N} \sum_{i=1}^{N} M(i)_{-} F C W_{j}(j=1,2,3, \ldots, N)
\end{gathered}
$$

For example, if reaction category $j$ of $F C W$ is recognized as positive and $M_{\text {mean }} F C W_{j}$ is large-valued, it implies that the drivers generally tend to be positive to FCW.

The coefficient of variation $\left(M_{c v_{-}} L D W_{j}\right.$ and $\left.M_{c v_{-}} F C W_{j}\right)$ can be used to measure the difference among drivers. The coefficients of variation can be calculated as shown below:

$$
\begin{gathered}
M_{c v \_} L D W_{j}=\frac{M_{S D \_} L D W_{j}}{M_{\text {mean__ }} L D W_{j}}(j=1,2,3, \ldots, N) \\
M_{c v \_} F C W_{j}=\frac{M_{S D \_} F C W_{j}}{M_{\text {mean_ }} F C W_{j}}(j=1,2,3, \ldots, N)
\end{gathered}
$$

$M_{S D \_} L D W_{j}$ and $M_{S D}-F C W_{j}$ are the standard deviations that can be calculated as shown below:

$$
\begin{gathered}
M_{S D \_} L D W_{j}=\sqrt{\frac{\sum_{i=1}^{N}\left(M(i)_{-} L D W_{j}-M_{\text {mean_ }} L D W_{j}\right)}{N}}(j=1,2,3, \ldots, N) \\
M_{S D \_} F C W_{j}=\sqrt{\frac{\sum_{i=1}^{N}\left(M(i)_{-} F C W_{j}-M_{\text {mean }} F C W_{j}\right)}{N}}(j=1,2,3, \ldots, N)
\end{gathered}
$$

\section{Results}

\subsection{Result of GMM}

The feature vector $x$ corresponding to LDW and FCW is considered to be the classification index and input to the GMM classifier. The authors determine the number of clusters by observing the value of the likelihood function. On the one hand, the authors want the likelihood function to be as large as possible to preserve the distribution characteristics of the data. On the other hand, the authors hope that the number of clusters may be small enough to achieve better practicability. The variation of likelihood function values under different cluster numbers is shown in Figures 6 and 7. 


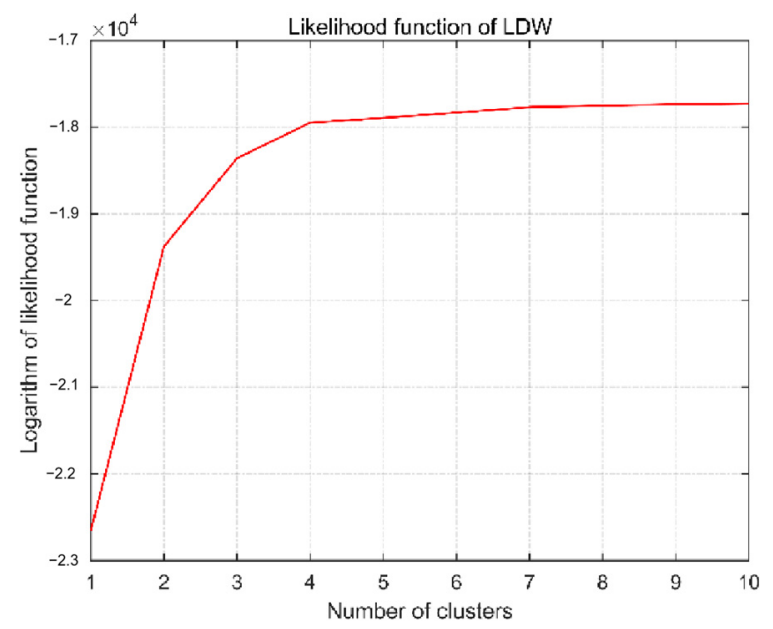

Figure 6. Likelihood function of LDW.

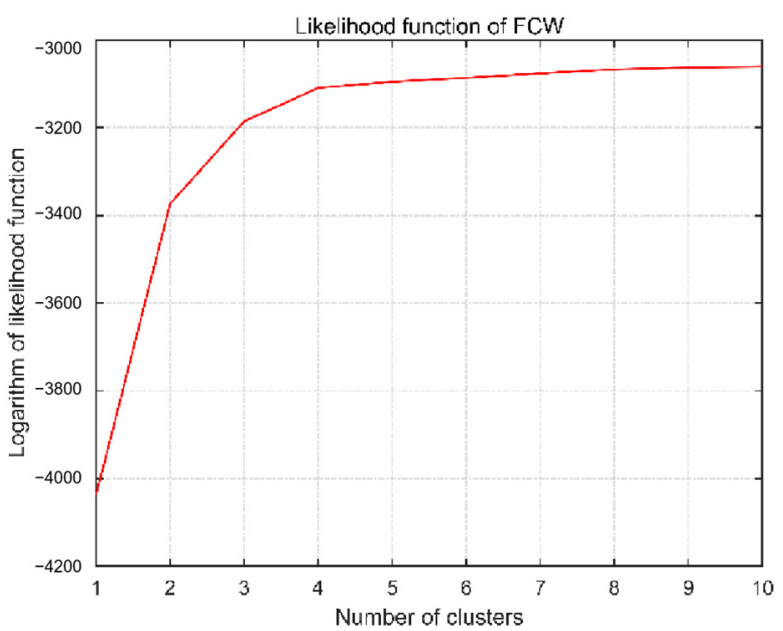

Figure 7. Likelihood function of FCW.

After the number of clusters reaches four, the change of likelihood function is relatively small. Therefore, in this study, the authors set the clustering category to four. The centers of each cluster in the initial state are selected based on the k-means method. After the EM algorithm iterates 114 steps (LDW) and 64 steps (FCW), every parameter converges. DS1, DS2, DS3, and DS4 are the four distributions. The classification parameters of the GMM classifier are listed in Table 1.

After obtaining the Gaussian mixture distribution, the corresponding region of each reaction category can be obtained as depicted in Figures 8 and 9. It is worth noting that the Gaussian mixture model used in this study is a classification method based on probability. There is actually no clear boundary between each category. The classification region of Figures 8 and 9 is determined by the maximum membership degree corresponding to the input. 
Table 1. Parameters of the Gaussian mixed model.

\begin{tabular}{|c|c|c|c|c|}
\hline Category & Mean & Covariance & e Matrix & Proportion \\
\hline LDW-DS1 & [ 5.8831 3.5787] & $\begin{array}{c}1.0502 \\
-0.0809\end{array}$ & $\begin{array}{c}-0.0809 \\
1.1844\end{array}$ & 0.2906 \\
\hline LDW-DS2 & {$\left[\begin{array}{ll}15.8273 & 3.4934\end{array}\right]$} & $\begin{array}{l}63.5913 \\
-0.1535\end{array}$ & $\begin{array}{c}-0.1535 \\
0.6317\end{array}$ & 0.2487 \\
\hline LDW-DS3 & [ 8.6936 11.2533] & $\begin{array}{l}11.2115 \\
-3.4531\end{array}$ & $\begin{array}{l}-3.4531 \\
37.5676\end{array}$ & 0.2694 \\
\hline LDW-DS4 & {$\left[\begin{array}{ll}51.0111 & 13.8568\end{array}\right]$} & $\begin{array}{c}0.00121 \\
-0.000202\end{array}$ & $\begin{array}{lc} & -0.000202 \\
2 & 0.000199\end{array}$ & 0.1913 \\
\hline FCW-DS1 & {$\left[\begin{array}{ll}-8.1035 & 3.0251\end{array}\right]$} & $\begin{array}{l}52.0797 \\
-2.1101\end{array}$ & $\left.\begin{array}{c}-2.1101 \\
1.5538\end{array}\right]$ & 0.4375 \\
\hline FCW-DS2 & {$\left[\begin{array}{lll}-16.2817 & 5.2704\end{array}\right]$} & $\begin{array}{c}127.5028 \\
4.7707\end{array}$ & $\begin{array}{l}4.7707 \\
2.2670\end{array}$ & 0.1388 \\
\hline FCW-DS3 & {$\left[\begin{array}{lll}-0.0106 & 4.1365\end{array}\right]$} & $\begin{array}{c}13.8499 \\
0.0105\end{array}$ & $\begin{array}{l}0.0105 \\
0.4118\end{array}$ & 0.3345 \\
\hline FCW-DS4 & {$\left[\begin{array}{ll}-2.2710 & 10.5774\end{array}\right]$} & $\begin{array}{c}46.2933 \\
-10.2554\end{array}$ & $\begin{array}{c}-10.2554 \\
19.5206\end{array}$ & 0.0792 \\
\hline
\end{tabular}

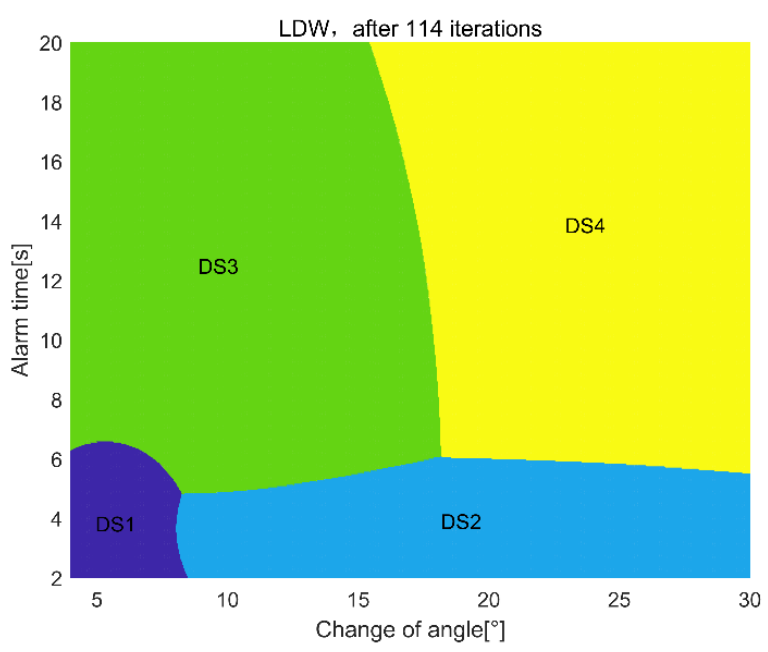

Figure 8. Classification result of reactions to LDW.

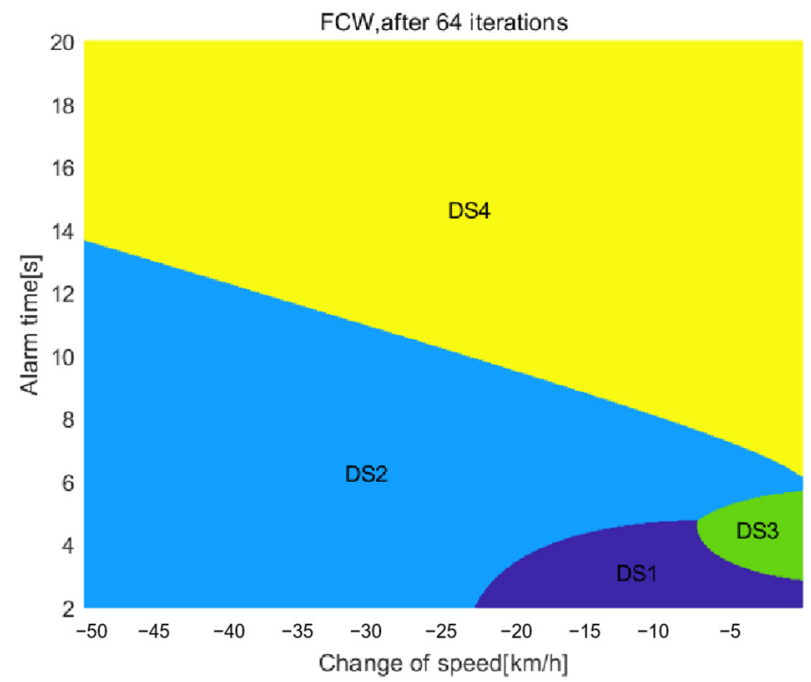

Figure 9. Classification result of reactions to FCW. 
From the above classification, the practical significance of the distributions are as follows.

LDW-DS1: In this type of warning, the warning duration is relatively short and the change in angle is relatively small $\left(<10^{\circ}\right)$. It means that drivers' response in this category is timely with a relatively small change in angle. This is a reasonable and safe response to the alarm and can be identified as positive reaction.

LDW-DS2: In this category, the drivers change their driving angle abruptly in a short time. According to our survey of videos recorded by buses, these events are mainly caused by drivers' overreaction or by a critical safety event. Considering that former research studies haven proven that crash rates increase as steering angle variation increases [41], such incidents should be avoided as much as possible.

LDW-DS3: In this category, although the angle that needs to be changed is relatively small, the response time of the drivers to LDW is relatively long. These events imply that the driver cannot respond to the alarm on time or they ignore the warning. This can be identified as a negative reaction and may influence driving safety.

LDW-DS4: In this category, alarm time is generally long and the change of angle is relatively large. After the verification of naturalistic video records (as shown in Figure 10), it can be found that records in this category are mainly caused by drivers' non-standard driving behavior (i.e., changing lanes without using turning signal). These records will not be further discussed in this paper.

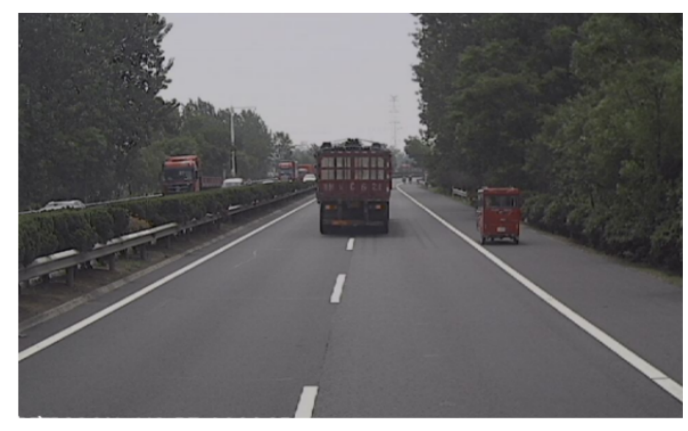

Figure 10. Changing lane without turning signal.

FCW-DS1: In this type of warning, the warning duration is relatively short and the change in vehicle speed is relatively obvious. These records are mainly caused by unreasonable following behavior. In this category, drivers take timely and appropriate speed deceleration. This is a reasonable and safe response to the alarm and can be considered as a positive reaction.

FCW-DS2: In this type of warning reaction, drivers often reduce the speed abruptly. These events are mainly caused by drivers' overreaction or by a critical safety event. They may lead to crashes because crash rates increase as speed variation increases $[39,40]$.

FCW-DS3: In this category, the alarm time is relatively long ( $>3 \mathrm{~s})$ and the change in speed is less obvious, implying that the driver has not paid enough attention to the warning. This category can be seen as negative reaction.

FCW-DS4: In this category, the alarm time is relatively long. After checking the original video records, these records are caused by irregular driving behaviors and are actually not near-crash situations (i.e., overtaking with a small headway). These records will not be further discussed in this paper.

Based on the above discussion, for either LDW or FCW, DS1 can be identified as positive reaction, DS2 as overreaction, and DS3 as negative reaction or emergency.

\subsection{Result of Drivers' Membership Degree to Reaction Types}

LDW-DS4 and FCW-DS4 are influenced by the abnormal data caused by incorrect detections and abnormal behaviors (such as lane changing without turning signal). In this 
paper, the authors describe the drivers' reactions by calculating their membership degree to DS1, DS2, and DS3.

The membership degrees of all the drivers are calculated using Equations (20) and (21). Figures 11 and 12 depict the membership degree of each driver that can be used to identify their typical reaction to warnings. Table 2 presents the statistical characteristics of the drivers' membership degrees.

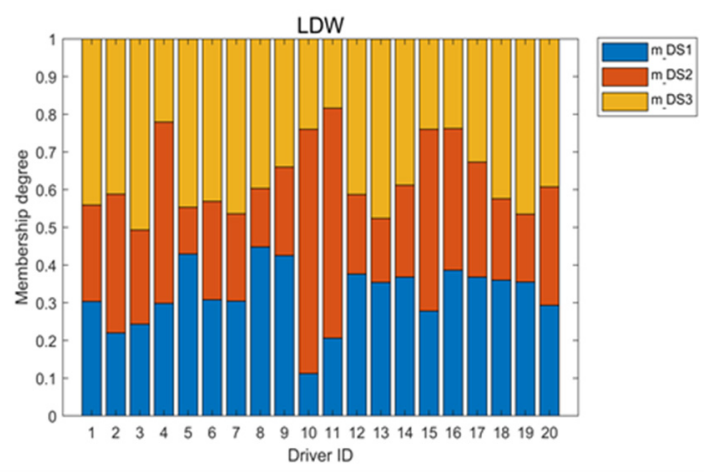

Figure 11. Membership of reactions to LDW.

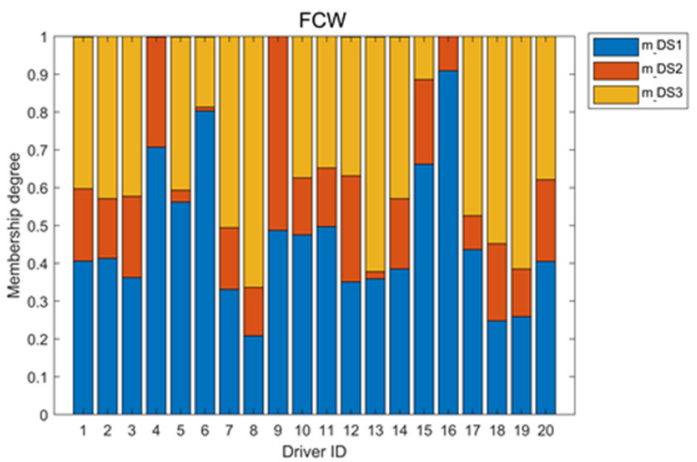

Figure 12. Membership of reactions to FCW.

Table 2. Statistical characteristics of drivers' membership degrees.

\begin{tabular}{|c|c|c|c|c|c|}
\hline \multirow{2}{*}{$\begin{array}{c}\text { Statistical } \\
\text { Characteristics }\end{array}$} & \multirow{2}{*}{ Type } & \multicolumn{2}{|c|}{ LDW } & \multicolumn{2}{|c|}{ FCW } \\
\hline & & $M_{m e a n \_} L D W_{j}$ & $M_{c v \_} L D W_{j}$ & $M_{m e a n \_} F C W_{j}$ & $M_{c v \_} F C W_{j}$ \\
\hline$j=1$ & positive & $38.7 \%$ & $22.3 \%$ & $52.8 \%$ & $36.0 \%$ \\
\hline$j=2$ & $\begin{array}{l}\text { overreaction } \\
\text { or emergency }\end{array}$ & $28.9 \%$ & $42.0 \%$ & $21.5 \%$ & $78.9 \%$ \\
\hline$j=3$ & negative & $32.4 \%$ & $30.4 \%$ & $25.7 \%$ & $71.5 \%$ \\
\hline
\end{tabular}

It is evident that, in general, the membership degrees of FCW to positive reactions are higher than those of LDW, revealing that the drivers' reactions to FCW are more positive overall. This finding supports the previous questionnaire survey regarding the acceptance of LDW and FCW [27]. By comparing the coefficients of variation, it can be concluded that the difference in the drivers' reactions to FCW is more obvious than to LDW. It depicts that the difference in drivers' acceptance of FCW is greater than that of LDW.

\subsection{Drivers' Membership Degree to Reaction Types in Different Environments}

By matching the road type with the alarm GPS information, the drivers' responses to the ADAS alarm on different types of roads through the drivers' naturalistic driving data was studied. 
From Figure 13, it can be observed that the bus drivers tend to be overreactive and ignorant to both LDW and FCW on urban roads, while they react more positively on freeways. This conclusion is consistent with the previous findings of car drivers through FOT [27].

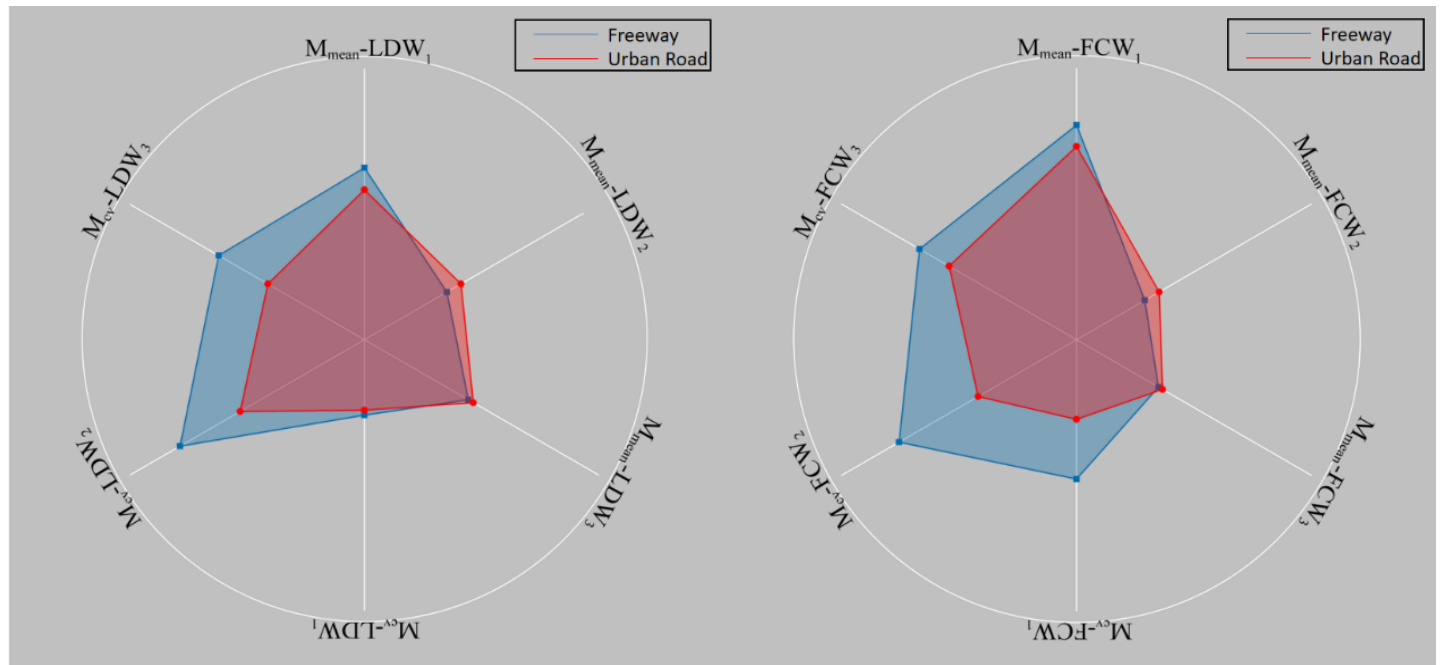

Figure 13. Statistical characteristics of drivers' membership degrees to different reaction types in freeways and urban roads.

In addition, the difference in the drivers' reactions to LDW and FCW is more evident in freeways. This shows that the reactions of drivers to the ADAS alarm are closer on urban roads than on freeways. This may be attributed to the fact that the constraints of a complex urban environment and traffic flows lead to increased forced lane departures and forward collision tendencies.

In actual driving, another important driving-environment change is the time of day. There are significant differences in traffic flow characteristics and the visibility between daytime and nighttime. The authors set 6:00 to 18:00 as daytime and 18:00 to 6:00 the next day as nighttime. Figure 14 illustrates the characteristics of the drivers' reactions during daytime and nighttime. Bus drivers behave more positively to LDW and FDW at night, particularly to FCW. This may be caused by the drivers' high mental workload at night [47].

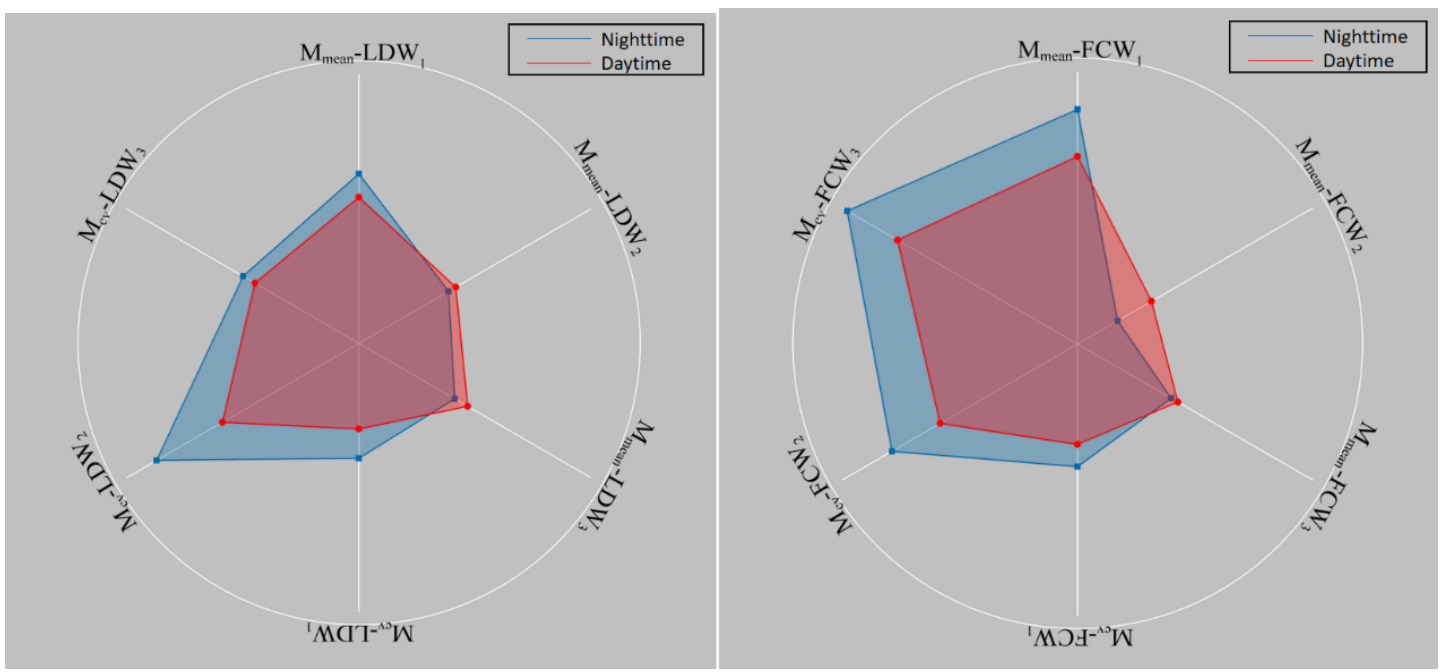

Figure 14. Statistical characteristics of drivers' membership degrees to the different reaction types during daytime and nighttime. 
The coefficient of variation of membership also has obvious differences during different times. The coefficients of variation are higher at night both for LDW and FCW. This implies that bus drivers' reaction shows greater difference at night.

\section{Discussion}

Previous studies have shown that the acceptance of the ADAS warning varies between drivers and the same driver's response to the ADAS warning in different environments also varies [48]. These differences have been investigated by various researchers using FOTs and driving simulator experiments by a control group or Likert scale [21,24,25,27,34-36]. However, there are still some gaps in former studies. The previous research objects are mainly private car drivers and it is uncertain whether the results apply to bus drivers. Moreover, some research methods based on driving experiments (especially the driving simulator experiments) encounter challenges [26]. Using naturalistic driving data of bus drivers for research provides a feasible solution to these problems [32]. In this study, the unsupervised learning method were employed to address bus drivers' naturalistic data.

Based on naturalistic driving data, it was found that the bus drivers responded more positively to FCW than to LDW and more positively to alarms on freeways than on urban roads, and this conclusion is consistent with previous studies of car drivers. [21,27]. The authors also found that drivers responded more positively to alarms on freeways than on urban roads and more positively to alarms at night than at day, which expanded the understanding of bus drivers' acceptance to ADAS.

Furthermore, by comparing the membership degree's coefficients of variation, the authors draw the conclusion that the difference in drivers' acceptance of FCW is greater than that of LDW and the difference in drivers' acceptance to both LDW and FCW is greater on the freeway than on urban roads, notably greater at night than during daytime. This study shows that LDW has a problem regarding the low acceptance and FCW has a problem regarding the large difference in bus drivers' acceptance. In addition, these problems may become more severe in specific environments. This study inspires designers to adjust the bus ADAS for different time periods and road environments.

This study also provides a new approach for the improvement of the ADAS. In this study, drivers' reactions were categorized into three types: negative, overreaction or emergency, and positive. Negative reactions reduce the effect of the ADAS warning system and may increase the drivers' driving risk. Overreaction (such as emergency braking and rapid lane change) may affect the operation of other vehicles nearby [39-41]. The developer of ADAS can reasonably change the alarm threshold according to the drivers' response characteristics to improve the acceptance of the ADAS alarm and develop adaptive ADAS to different drivers. Previous studies have designed ADAS alarm thresholds adaptively based on drivers' car-following characteristic parameters [49]. In this study, new characteristic parameters based on bus drivers' reactions are proposed by the Gaussian mixed model and fuzzy logic method, which can be seen as the principle of the ADAS design.

However, although this experiment can determine the differences in drivers' responses to the alarm in different environments, it cannot obtain the specific reasons for these differences. This may hinder the improvement of the ADAS. For example, in urban roads, the drivers' responses to the alarm are less positive. One possible explanation is that the relatively complex driving environment of urban roads increases the drivers' cognitive dispersion [50]. Another explanation is that the complex traffic environment on urban roads encourages compulsive behaviors in drivers. The accuracy of FCW and LDW may decrease due to complex traffic conditions, thereby affecting the drivers' trust in the system. Therefore, it is required to appropriately reduce the difference in the environment and test the drivers' cognitive ability (by rapid serial visual presentation (RSVP) [51] or mental math) in further studies.

Moreover, there exists another limitation in this study. When discussing DS3, the authors do not distinguish between emergency and overreaction, and give an explanation based on security performance. However, from the perspective of drivers' acceptance of 
ADAS, it is necessary to distinguish the two behaviors. Some detection methods based on computer vision may be needed to solve this problem completely.

Author Contributions: Conceptualization, W.Y. and Y.X.; data curation, W.Y. and F.Z.; formal analysis, W.Y.; funding acquisition, Z.Y.; methodology, W.Y. and X.S.; resources, Z.Y.; validation, W.Y.; writing—original draft preparation, W.Y.; writing—review and editing, Y.X. and F.Z. All authors have read and agreed to the published version of the manuscript.

Funding: This study was sponsored by the National Natural Science Foundation of China (number 52072070), the Foundation for Jiangsu Key Laboratory of Traffic and Transportation Security (TTS202004), and the Fundamental Research Funds for the Central Universities (number 2242021R10112).

Institutional Review Board Statement: Not applicable.

Informed Consent Statement: Informed consent was obtained from all subjects involved in the study. Data Availability Statement: Not applicable.

Acknowledgments: Authors thank Jiangsu Yudao Data Technical Limited Company for the support of commercial vehicles' operational and forward collision warning data.

Conflicts of Interest: The authors declare no conflict of interest.

\section{References}

1. World Health Organization. Global Status Report on Road Safety 2018; World Health Organization: Geneva, Switzerland, 2018.

2. Gao, Y.; Deng, Y.; Wang, X. Traffic Safety Situation and Countermeasures for City Bus Passenger Transportation. China Public Security Acad. Ed. 2015, 2, 1-5.

3. Guo, Y.; Sayed, T.; Essa, M. Real-time conflict-based Bayesian Tobit models for safety evaluation of signalized intersections. Accid. Anal. Prev. 2020, 144, 105660. [CrossRef]

4. Li, D.; Song, Y.; Sze, N.N.; Li, Y.; Miwa, T.; Yamamoto, T. An alternative closed-form crash severity model with the non-identical, heavy-tailed, and asymmetric properties. Accid. Anal. Prev. 2021, 158, 106192. [CrossRef] [PubMed]

5. Li, M.; Li, Z.; Xu, C.; Liu, T. Short-term prediction of safety and operation impacts of lane changes in oscillations with empirical vehicle trajectories. Accid. Anal. Prev. 2020, 135, 105345. [CrossRef] [PubMed]

6. Wang, C.; Xie, Y.; Huang, H.; Liu, P. A review of surrogate safety measures and their applications in connected and automated vehicles safety modeling. Accid. Anal. Prev. 2021, 157, 106157. [CrossRef]

7. Treat, J.R.; Tumbas, N.S.; McDonald, S.T.; Shinar, D.; Hume, R.D.; Mayer, R.E. Tri-level study of the causes of traffic accidents (DOT-HS-034-3-535-77-TAC).

8. Corona, D.; De Schutter, B. Adaptive cruise control for a SMART car: A comparison benchmark for MPC-PWA control methods. IEEE Trans. Control Syst. Technol. 2008, 16, 365-372. [CrossRef]

9. Bond, J.V., III; Engelman, G.H.; Ekmark, J.; Jansson, J.L.; Tarabishy, M.N.; Tellis, L. Driver Augmented Autonomous Braking System. U.S. Patent 6,517,172, 11 February 2003.

10. Jo, J.; Lee, S.L.; Kim, J.; Jung, H.G.; Park, K.R. Vision-based method for detecting driver drowsiness and distraction in driver monitoring system. Opt. Eng. 2011, 50, 127202. [CrossRef]

11. Srinivasa, N. Vision-based vehicle detection and tracking method for forward collision warning in automobiles. In Proceedings of the IEEE Intelligent Vehicle Symposium, Versailles, France, 17-21 June 2002. [CrossRef]

12. Zheng, Y.; Ran, B.; Qu, X.; Zhang, J.; Lin, Y. Cooperative lane changing strategies to improve traffic operation and safety nearby freeway off-ramps in a connected and automated vehicles environment. IEEE Trans. Intel. Trans. Syst. 2019, 21, 4605-4614. [CrossRef]

13. Birrell, S.A.; Fowkes, M.; Jennings, P.A. Effect of using an in-vehicle smart driving aid on real-world driver performance. IEEE Trans. Intel. Trans. Syst. 2014, 15, 1801-1810. [CrossRef]

14. Remboski, D.; Gardner, J.; Wheatley, D.; Hurwitz, J.; Mactavish, T.; Gardner, R.M. Driver Performance Improvement through the Driver Advocate: A Research Initiative toward Automotive Safety. In Proceedings of the Convergence 2000 International Congress on Transportation Electronics; 2000; pp. 509-518.

15. Keller, A. The Future of Motor Insurance: How Car Connectivity and ADAS Are Impacting the Market; Joint Whitepaper by HERE and Swiss, R: Zurich, Switzerland, 2016.

16. Alkim, T.P.; Bootsma, G.; Hoogendoorn, S.P. Field operational test "The Assisted Driver". 2007 Intell. Veh. Symp. 2007. [CrossRef]

17. Hagl, M.; Kouabenan, D.R. Safe on the road-Does advanced driver-assistance systems use affect road risk perception? Transp. Res. Part F Traffic Psychol. Behav. 2020, 73, 488-498. [CrossRef]

18. Weiner, E. Volvo Developing Driver-Monitoring Sensors. 2014. Available online: http://www.automobilemag.com/features/ news / 1403-volvo-driver-state-estimationmonitoring-sensors (accessed on 20 March 2021).

19. Rudin-Brown, C.M.; Noy, Y.I. Investigation of behavioral adaptation to lane departure warnings. Transp. Res. Rec. 2002, 1803, 30-37. [CrossRef] 
20. Cades, D.M.; Crump, C.; Lester, B.D.; Young, D. Driver distraction and advanced vehicle assistive systems (ADAS): Investigating effects on driver behavior. Adv. Intell. Syst. Comput. 2017, 484, 1015-1022. [CrossRef]

21. Son, J.; Park, M.; Park, B.B. The effect of age, gender and roadway environment on the acceptance and effectiveness of Advanced Driver Assistance Systems. Transp. Res. Part F Traffic Psychol. Behav. 2015, 31, 12-24. [CrossRef]

22. Winsum, W.V.; Heino, A. Choice of time-headway in car-following and the role of time-to-collision information in braking. Ergonomics 1996, 39, 579-592. [CrossRef]

23. Reymond, G.; Kemeny, A.; Droulez, J.; Berthoz, A. Role of lateral acceleration in curve driving: Driver model and experiments on a real vehicle and a driving simulator. Human Fact. 2001, 43, 483-495. [CrossRef]

24. Saito, Y.; Itoh, M.; Inagaki, T. Driver assistance system with a dual control scheme: Effectiveness of identifying driver drowsiness and preventing lane departure accidents. IEEE Trans. Human-Mach. Syst. 2016, 46, 660-671. [CrossRef]

25. Maag, C.; Mühlbacher, D.; Mark, C.; Krüger, H.P. Studying effects of Advanced Driver Assistance Systems (ADAS) on individual and group level using multi-driver simulation. In Proceedings of the 2011 IEEE Intelligent Vehicles Symposium (IV), Baden-Baden, Germany, 5-9 June 2011. [CrossRef]

26. Groeger, J.A.; Murphy, G. Driver performance under simulated and actual driving conditions: Validity and orthogonality. Accid. Anal. Prev. 2020, 143, 105593. [CrossRef]

27. Lyu, N.; Deng, C.; Xie, L.; Wu, C.; Duan, Z. A field operational test in China: Exploring the effect of an advanced driver assistance system on driving performance and braking behavior. Transp. Res. Part F Traffic Psychol. Behav. 2019, 65, 730-747. [CrossRef]

28. Mullen, N.; Charlton, J.; Devlin, A.; Bédard, M. Simulatorvalidity: Behaviors observed on the simulator and on the Road. In Handbook of Driving Simulation for Engineering, Medicine, and Psychology; CRC Press: Boca Raton, FL, USA, 2011.

29. Eby, D.W.; Silverstein, N.M.; Molnar, L.J.; Leblanc, D.; Adler, G. Driving behaviors in early stage dementia: A study using in-vehicle technology. Accid. Anal. Prev. 2012, 49, 330-337. [CrossRef]

30. Ellison, A.B.; Greaves, S.P.; Bliemer, M.C.J. Driver behaviour profiles for road safety analysis. Accid. Anal. Prev. 2015, 76, 118-132. [CrossRef]

31. Mahapatra, G.; Maurya, A.K. Study of vehicles lateral movement in non-lane discipline traffic stream on a straight road. Procedia Soc. Behav. Sci. 2013, 104, 352-359. [CrossRef]

32. Schagen, I.V.; Sagberg, F. The Potential Benefits of Naturalistic Driving for Road Safety Research: Theoretical and Empirical Considerations and Challenges for the Future. Procedia Soc. Behav. Sci. 2012, 48, 692-701. [CrossRef]

33. Orlovska, J.; Novakazi, F.; Lars-Ola, B.; Karlsson, M.; Wickman, C.; Söderberg, R. Effects of the driving context on the usage of Automated Driver Assistance Systems (ADAS) - Naturalistic Driving Study for ADAS evaluation. Transp. Res. Interdiscip. Perspect. 2020, 4, 100093. [CrossRef]

34. Li, G.; Li, S.E.; Cheng, B. Field operational test of advanced driver assistance systems in typical Chinese road conditions: The influence of driver gender, age and aggression. Int. J. Automot. Technol. 2015, 16, 739-750. [CrossRef]

35. Blaschke, C.; Breyer, F.; Färber, B.; Freyer, J.; Limbacher, R. Driver distraction based lane-keeping assistance. Transp. Res. Part F Traffic Psychol. Behav. 2009, 12, 288-299. [CrossRef]

36. Butakov, V.; Ioannou, P. Personalized Driver/Vehicle Lane Change Models for ADAS. IEEE Trans. Veh. Technol. 2015, 64, 4422-4431. [CrossRef]

37. Sayer, J.; LeBlanc, D.; Bogard, S.; Funkhouser, D.; Bao, S.; Buonarosa, M.L.; Blankespoor, A. Integrated Vehicle-Based Safety Systems Field Operational Test Final Program Report; U.S. Department of Transportation: Ann Arbor, ML, USA, 2011.

38. Xiong, H.; Boyle, L.N.; Moeckli, J.; Dow, B.R.; Brown, T.L. Use patterns among early adopters of adaptive cruise control. Hum. Factors 2012, 54, 722-733. [CrossRef]

39. Aarts, L.; Schagen, I.V. Driving speed and the risk of road crashes: A review. Accid. Anal. Prev. 2006, 38, 215-224. [CrossRef]

40. Garber, N.J.; Gadiraju, R. Factors Affecting Speed Variance and its Influence on Accidents. Transp. Res. Rec. J. Transp. Res. Board 1989, 1213, 64-71.

41. Park, S.; Ritchie, S.G. Exploring the Relationship among Freeway Speed Variance, Lane Changing, and Vehicle Heterogeneity. Presented at 83rd Annual Meeting of the Transportation Research Board, Washington, DC, USA, 11-15 January 2004.

42. Mohammadnazar, A.; Arvin, R.; Khattak, A.J. Classifying travelers' driving style using basic safety messages generated by connected vehicles: Application of unsupervised machine learning. Transp. Res. Part C Emerg. Technol. 2021, $122,102917$. [CrossRef]

43. Qi, G.; Wu, J.; Zhou, Y.; Du, Y.; Jia, Y.; Hounsell, N.; Stanton, N.A. Recognizing driving styles based on topic models. Transp. Res. Part D Transp. Environ. 2019, 66, 13-22. [CrossRef]

44. Wang, W.; Xi, J.; Chong, A.; Li, L. Driving style classification using a semisupervised support vector machine. IEEE Trans. Human-Mach. Syst. 2017, 47, 650-660. [CrossRef]

45. Eftekhari, H.R.; Ghatee, M. Hybrid of discrete wavelet transform and adaptive neuro fuzzy inference system for overall driving behavior recognition. Transp. Res. Part F Traffic Psychol. Behav. 2018, 58, 782-796. [CrossRef]

46. Vasconez, J.P.; Viscaino, M.; Guevara, L.; Cheein, F.A. A fuzzy-based driver assistance system using human cognitive parameters and driving style information. Cogn. Syst. Res. 2020, 64, 174-190. [CrossRef]

47. Brookhuis, K.A.; van Driel, C.J.G.; Hof, T.; van Arem, B.; Hoedemaeker, M. Driving with a congestion assistant; mental workload and acceptance. Appl. Ergon. 2009, 40, 1019-1025. [CrossRef] [PubMed] 
48. Liang, Y.; McLaurin, E.J.; Simmons, L.A.; Verma, S.K.; Horrey, W.J.; Lesch, M.F. Considering Traffic and Roadway Context in Driver Behavior Assessments: A Preliminary Analysis. In Proceedings of the 8th International Conferenceon Automotive User Interfaces and Interactive Vehicular Applications, Ann Arbor, MI, USA, 24-26 October 2016.

49. Wang, J.; Yu, C.; Li, S.E.; Wang, L. A Forward Collision Warning Algorithm with Adaptation to Driver Behaviors. IEEE Trans. Intell. Transp. Syst. 2015, 17, 1157-1167. [CrossRef]

50. Son, J.; Lee, Y.; Kim, M.H. Impact of traffic environment and cognitive workload on older drivers' behavior in simulated driving. Int. J. Precis. Eng. Manuf. 2011, 12, 135-141. [CrossRef]

51. Reinmueller, K.; Steinhauser, M. Adaptive forward collision warnings: The impact of imperfect technology on behavioral adaptation, warning effectiveness and acceptance. Accid. Anal. Prev. 2019, 128, 217-229. [CrossRef] 